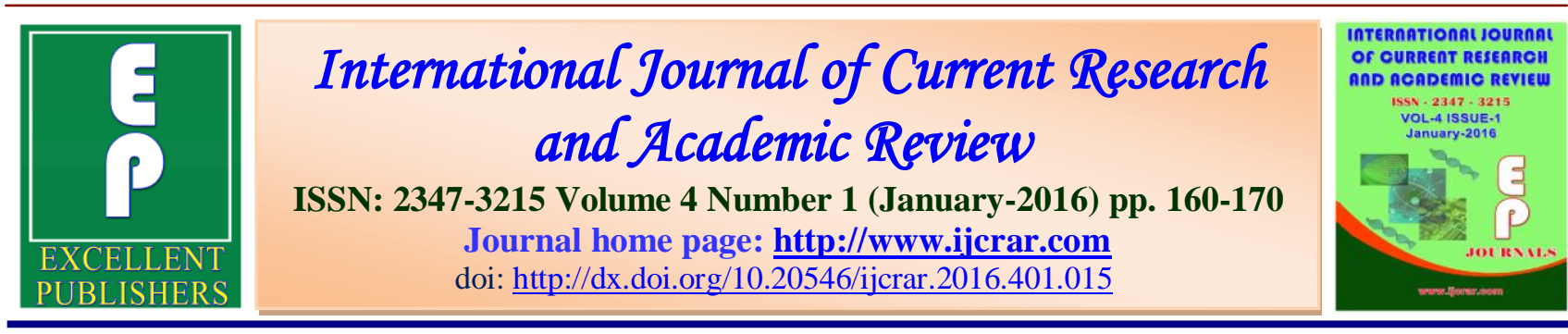

\title{
Streptococcal Dental Caries - A Short Review
}

\section{M.D. Dinesh ${ }^{1}$, M.S. Uma ${ }^{1}$, S. Meenatchisundaram ${ }^{1}$, V.M. Anjali ${ }^{1}$, P.S. Athira ${ }^{2}$ and Assitha Carmel $^{3}$}

${ }^{1}$ Department of Microbiology, Nehru Arts and Science College, Coimbatore, India

${ }^{2}$ Department of Microbiology, Pazhassiraja College, Pulpally, Wayanad, India

${ }^{3}$ Department of Microbiology, Mar Athanasious College, Kothamangalam, Ernakulam, India

\section{*Corresponding author}

\begin{tabular}{|c|c|}
\hline KEYWORDS & $A B S T R A C T$ \\
\hline $\begin{array}{l}\text { Dental caries, } \\
\text { S. mitis, } \\
\text { S.mutans, } \\
\text { S.gordonii, } \\
\text { aetiology }\end{array}$ & $\begin{array}{l}\text { Dental caries is a permanent infection usually bacterial in cause today; caries } \\
\text { remains one of the most common diseases throughout the world. Cardiology } \\
\text { is the study of dental caries. Dental caries is important yet a preventable } \\
\text { public health problem. It is the most universal chronic disease of childhood } \\
\text { that interferes with usual nutrition intake, speech, self-esteem and daily } \\
\text { regular activities, because the caries pain adversely affects the normal food } \\
\text { intake. This review article provides a summary of the development of dental } \\
\text { caries, role in microbial communities, aetiology, Prevention etc. }\end{array}$ \\
\hline
\end{tabular}

\section{Introduction}

Dental caries is an infectious disease of the teeth that results in localized dissolution and destruction of the calcified tissues. It is considered to be a disease of civilized society, related to lifestyle factors, but heredity also plays a role. Dental caries is a multifactorial human disease that has widely effected many populations all over the world. This is caused by accumulation of biofilm on tooth surface (1). Manifestations of the disease occur when there is an imbalance between the biofilm and the host due to changes in biofilm matrix $\mathrm{pH}$ caused by diet, microorganisms, or salivary flow and their components (2).
Dental plaque is a general term for the diverse microbial community (predominantly bacteria) found on the tooth surface, embedded in a matrix of polymers of bacterial and salivary origin. Dental caries will not occur if the oral cavity is free of bacteria. These bacteria are organized into a material known as dental plaque which is yellowish colored film on the surface of the teeth. Of the many types of bacteria in the mouth, the most caries active appear to be Streptococcus mutans, Streptococcus mitis, Lactobacillus sp., Veillonella sp. and Actinomyces sp. These bacteria can be transferred from mother to 
child and are present at varying levels in all human mouths. A variety of carbohydrates provide substrates for these organisms to grow on and the waste products of their metabolism - acids - initiate the tooth decay process by dissolving tooth enamel.

\section{Viridans Group Streptococci}

Viridans group streptococci (VGS) are inhabitants of the oral cavity, which is sterile at birth, but at the age of one year, Streptococcus species compose $70 \%$ of the cultivable oral flora and S.salivarius, S.oralis and S.mitis are predominant. VGS are also found in the gastrointestinal and urogential tracts. The term viridans is derived from the Latin viridis or green, and refer to the sheen caused by haemolysis (Alpha- Haemolysis) around the bacterial colonies on blood agar. Viridans group streptococci are divided into five major groups, which affect humans (Table 1).

\section{Streptococcal Dental Caries}

Streptococcus mutans is first described by Clark in 1924 after he isolated it from a carious lesion after that the real interest in this microbe was developed when researchers began studying dental caries in detail. Researchers have identified several hundred genes that appear to be unique to the organism. These are potential drug targets because disrupting them would disable the pathogen without harming the other bacteria in the mouth (4).

\section{Streptococcus mitis}

Streptococcus mitis are commensal bacteria and belongs to the viridans streptococci group that colonize hard surfaces in the oral cavity such as dental hard tissues as well as mucous membranes and are part of the oral flora. They are usually arranged in short chains in the shape of cocci (5). These Gram-positive bacteria are not usually pathogenic but commonly cause bacterial endocarditis, which is the inflammation of an inner layer of the heart. S. mitis are alpha hemolytic, meaning it can break down red blood cells. S. mitis are not motile, do not form spores and lack group-specific antigens. S. mitis cells grow in pairs or short chains and they present an arrow-headed shape with a length of $0.5 \mu \mathrm{m}$ (6). $S$. mitis live optimally at temperatures between $30^{\circ} \mathrm{C}$ and $35^{\circ} \mathrm{C}$, making them mesophiles. They are facultative anaerobes, which is a bacterium that makes ATP by aerobic respiration if oxygen is present but is also capable of switching to fermentation in the absence of oxygen (7).

The adherence and colonization by Streptococcus mitis can be summarized into four steps: the first two steps involve (A) the long range interaction adherence with suitable surface molecules through pili bacteria, and (B) the short range adherence of some pioneers bacteria which forms stronger bonds with the surface molecules leading to multiple adhesins. During the third step $(\mathrm{C})$, the intermicrobial signalling, the formation of extracellular polymeric substance (EPS) and the nutritional adaption, give rise to a society produced by cell division and multiplication. In the last step (D) the incorporation of other microorganisms together with cell-cell signaling, intergenic coaggregation, metabolic synergy and genetic exchange, leads to the formation of a community (8). The development of the community is dependent on many factors including adherence, signalling, nutritional adaption, and host modulation. In addition, environmental conditions such as $\mathrm{pH}$, temperature, oxygen availability, organic metabolites etc. may influence Streptococcus mitis colonization. 


\section{Streptococuus mutans}

S. mutans is a significant pathogen of oral cavity and initiates dental caries. This organism was isolated for the first time from the dental plaque by Clarke in 1924 (9). The name 'mutans' was chosen because of its tendency to exhibit both coccal and rod shaped (mutant) cell morphology (10). S.mutans belongs to the $S$ viridans group, which is a poorly defined, heterologous group of the genus Streptococcus. Members of this group are indigenous to the oral cavity. Though the classification of this particular group is oversimplified, it includes a variety of strains with different physiological and serological characteristics comprising $S$. mutans group, $S$. sanguis group, S. mitis group, $S$. salivarius group and $S$. milleri group (11). Many factors contribute to the cariogenicity of this organism such as rapid generation time, fermentation of wide range of carbohydrates, ability to withstand low $\mathrm{pH}$, presence of enzyme glucosyl transferase that converts sucrose to glucose to enhance adhesion (10).

In humans, Streptococcus mutans and Streptococcus sobrinus, are the most prevalent cariogenic bacteria (12). A large number of studies support the strong association between mutans Streptococci level and the incidence of carious lesions (13). The presence of mutans Streptococci, both in dental plaque and saliva of young caries-free children, appears to be associated with an increase in caries risk (14). Early acquisition of mutans Streptococci is also a major risk factor of early childhood caries and the development of future caries (15). S. mutans has been the most intensely investigated cariogenic bacterium in the literature, because of its strong correlation with dental caries and the relatively low presence without disease. This bacterium, which is considered "a specialist" in causing dental caries, possesses multiple inherent virulence properties essential for its cariogenic potential.

Acidogenicity, acid tolerance, adhesion and biofilm formation are considered the main virulence properties associated with the cariogenicity of $S$. mutans $(16,17) S$. mutans is capable of metabolizing a wide variety of sugars to lactic acids. The acids produced not only are responsible for tooth demineralization but also rapidly acidify the dental plaque environment around the bacterium. While $S$. mutans can adapt to the surrounding $\mathrm{pH}$ as low as $\mathrm{pH} 4.4$, this vastly acidic condition is toxic to most other bacteria. The adhesion of $S$. mutans to tooth surfaces is mediated by a two-step process, involving sucrose-independent and sucrosedependent mechanisms. Sucroseindependent adhesion to salivary pellicle is believed to initiate the attachment process, whereas sucrose-dependent adhesion may enhance bacterial colonization to the tooth surface $(16,17)$.

Biofilm formation is an important virulence factor for $S$. mutans. The bacteria must be able to adhere to be maintained in the oral cavity $(18,19)$ found there was no association between the levels of mutans streptococci in the saliva and caries status but there was an association between the amount of biofilm-forming mutans streptococci and caries incidence. When cells are growing in a biofilm bacterial susceptibility to antimicrobials is often reduced (20-22) and in established biofilms the antimicrobial effect is limited to the superficial layers (23). Sucrose is extremely important in $S$. mutans biofilm formation. The presence of sucrose decreases the antimicrobial susceptibility of $S$. mutans, probably due to the subsequent biofilm formation (24) initially, or in the absence 
of sucrose, S. mutans adheres using ionic and lectin-like interactions. Streptococcal protein antigen $\mathrm{P}$ binds to salivary agglutinin glycoprotein (SAG) and SAG coated surfaces. In the presence of sucrose, glucans are synthesised by glucosyltransferases (GTFs). They form an extracellular layer of EPS that promote adhesion and biofilm formation (25).

\section{Streptococcus gordonii}

S. gordonii is part of the group viridians of Streptococci, nonpathogenic commensal streptococci, which are integral members of the human oral flora. These organisms colonize tooth surfaces by creating biofilms in the human mouth, also known as dental plaque. S.gordonii plays an integral role in initiating colonization by creating surfaces for other colonizers to adhere to. $S$. gordonii also causes bacterial endocarditis by entering the blood stream usually after oral trauma. S. gordonii colonizes plateletfibrin thrombi, blood clotting agents, in damaged heart valves or endocardium leading to damage and dysfunction of the heart valves. Endocarditis can be treated with antibiotic therapy and may cause death (The Health Central Network, Inc.).

Initially, Streptococcus gordonii initiates colonization through formation of a monospecies biofilm. The human tooth is covered by pellicle containing lipids and proteins, including salivary agglutinin glycoprotein. The receptors for salivary agglutin glycoprotein located on $S$. gordonii and other pioneer colonizers recognize and bind to the pellicle. $S$. gordonii cells, bound to the surface of the tooth, and then initiate a signal transduction pathway, known as BrfAB, which regulates adhesive activity. For current research Streptococcus gordonii is good candidate for a "live bacterial mucosal vaccine vector."
The efficiency of $S$. gordonii in colonizing the oral cavity creates great potential for the stimulation of the mucosal immune system. S. gordonii can also be easily controlled genetically to produce a "fusion construct" that will enhance its stability. Another advantage is that heterologous antigens may be attached to the cell wall of $S$. gordonii thereby expressing a number of viral and bacterial antigens. Some examples of possible antigens are the E7 protein of human papillomavirus, the B subunit of Escherichia coli heat-labile toxin and the tetanus toxin fragment.

\section{Diet and Dental Caries}

Many factors influence caries development, including the presence of plaque-producing bacteria, innate susceptibility of tooth surfaces, frequency of eating, snacking behaviour, oral hygiene practices, fluoride availability, and salivary flow and composition. All carbohydrates, including starchy foods, especially highly processed starches in savoury snacks, and foods containing sugars such as cakes, biscuits, jam, honey, fruits and fruit juices can be used by bacteria to produce acids, which accelerate demineralisation of tooth enamel. Saliva contains protective minerals (calcium and phosphates) that buffer the bacterial acids and promote remineralisation. The greater the salivary flow, the more rapid the remineralisation. It is the balance between acid production and salivary recovery that determines susceptibility to caries.

Diet also plays a significant aetiological role in dental erosion, the prevalence of which seems to be increasing, and dietary components may contribute to development of enamel defects (e.g. enamel hypoplasia, fluorosis). According to Sheiham (2001), "sugars, particularly sucrose, are the most important dietary aetiological cause of caries 
and the main strategy to further reduce the levels of caries is reducing the frequency of sugars intakes in the diet". A sucrose-rich diet increases the growth rate of many oral bacteria and changes the composition of the microflora in a caries-promoting manner $(26,27)$.

Repeated acid attacks which do not give teeth the time to recover increase risk of caries, so small amounts of sugar and other fermentable carbohydrates eaten frequently during the day will increase caries risk more than large amounts eaten infrequently. Additionally, the amount of sugar consumed is less important than how quickly it is cleared from the mouth. Sticky foods like breads or raisins stay in the mouth much longer, and increase the potential for decay. Calcium rich foods like cheese, eaten immediately after sugar, can help protect against demineralisation. The relationship between the amount of sugar consumed and the levels of decay in individuals is actually very weak. Dietary methods of preventing caries have not been shown to be effective. The most effective means of preventing caries is routine use of fluoride toothpaste in conjunction with proper oral hygiene practices.

\section{Etiology of Dental Caries}

Dental caries is a chronic disease, a process that progresses very slowly in most individuals. Dental caries is a multifactorial disease that is caused by interplay of three major factors, i.e., teeth, cariogenic bacteria, and fermentable sugars. Epidemiological studies revealed the relationship between caries prevalence and sugar consumption. Dental caries is the infectious and transmissible disease caused by mutans streptococci. Mutans streptococci include several different species, including S.mutans and S.sobrinus, which are found in human caries. The most important virulence factor of mutans streptococci is glucosyltransferase(s) which synthesize adhesive, water-insoluble glucans from sucrose. $S$. mutans and $S$. sobrinus produce three and four glucosyltransferases, respectively, whose cooperative actions is essential for adhesive glucan synthesis. Adhesive glucans mediate attachment of bacteria to the tooth surface as well as to each other. As mutans streptococci require the tooth surface as a habitat, infants do not harbor this organism until sometime after the teeth emerge. The major source of mutans streptococci are their mothers. The detection rate of mutans streptococci increases with age. Dental plaque is a typical biofilm consisting of micro-organisms and their products such as adhesive glucan. Plaque bacteria produce a large quantity of acids such as lactic acid from fermentable carbohydrates. Acids are entrapped between the tooth surface and plaque biofilm, then the $\mathrm{pH}$ of the enamel easily falls below 5.6, and loss of mineral (decalcification) from enamel is induced. This is initiation of dental caries (22). The current concepts of dental caries focus on the fermentation of carbohydrates by cariogenic bacteria producing organic acids. Plaque bacteria produce a variety of end products that may differ depending on the diet. When fermentable carbohydrates are present, the main organic acids produced are lactic, formic, and acetic acids $(28,29)$. The use of tobacco may also increase the risk for caries formation. Smokeless tobacco frequently contains high sugar content in some brands, possibly increasing the susceptibility to caries.

\section{Sign and Symptoms of Dental Caries}

A person experiencing caries may not be aware of the disease. Pain due to dental caries can range from mild to severe depending on its progress. In some cases, if 
the decay progresses very slowly, pain may be totally absent too. The earliest sign of a new carious lesion is the appearance of a chalky white spot on the surface of the tooth, indicating an area of demineralization of enamel. This is referred to as incipient decay. As the lesion continues to dematerialize, it can turn brown but will eventually turn into a cavitations ("cavity"). Before the cavity forms, the process is reversible, but once a cavity forms, the lost tooth structure cannot be regenerated. A lesion that appears brown and shiny suggests dental caries were once present but the demineralization process has stopped, leaving a stain. A brown spot that is dull in appearance is probably a sign of active caries.

One of the signs of dental cavities, sensitivity to cold foods is an indication that the tooth is still alive. There is a possibility of saving the tooth with a filling if treated on time. However, if feel sensitivity and pain when consume anything hot, it may be a sign of a dying or dead tooth. Teeth chipping off when bite or chew on anything moderately hard; it may be a sign of dental decay. The way the enamel (outermost layer of the tooth) and dentine (the second layer of the tooth) are structured, when the decay progressed to dentin, it undermines the overlying enamel. This makes the enamel vulnerable to fractures. Darkening of teeth is a sign of dental decay. Basically, when the decay progresses to the pulp, there is a lot of destruction of the dentine. This coupled with the fact that the dying or dead pulp produces certain pigments makes the teeth look dark. When the dental decay reaches the pulp of the tooth and then progresses to the tissues surrounding it along with the bone, it generates pus. This can cause swelling in the gums and often has to be treated immediately. As the enamel and dentin are destroyed, the cavity becomes more noticeable. The affected areas of the tooth change color and become soft to the touch. Once the decay passes through enamel, the dentinal tubule, which have passages to the nerve of the tooth become exposed and causes a tooth ache. The pain may worsen with exposure to heat, cold, or sweet foods and drinks. Dental caries can also cause bad breath and foul tastes. In highly progressed cases, infection can spread from the tooth to the surrounding soft tissues. Complications such as cavernous sinus thrombosis and Ludwig's angina can be life-threatening.

\section{Epidemiology}

From an epidemiological point of view, dental caries is widely disseminated across the world and may be considered a public health problem in world wide. Dental diseases are one of the most common of non-communicable diseases. Though they are rarely life threatening, they do impact the quality of life. Thus, they are an important public health concern. According to the World Health Organization (WHO), the prevalent oral diseases are dental caries, periodonatal diseases and edentulousness.

Prevalence of dental caries is high and provision for restorative treatment is inadequate in most parts of the country. DCI's National survey is been conducted to determine the prevalence of dental diseases in different states of India. Prevalence of dental caries is $40 \%-80 \%$ (Very high in Northern states 85\%-90\%), Periodontal conditions usually increase with age and are found more in rural areas. Oral cancer and precancerous conditions are 3\%-10\% (Highest being in Orissa 7\%). In perspective of oral health, most of the Indian populace is affected with the common oral problems like periodontal disease being 90-95\% followed by dental caries affecting nearly $60-80 \%$ of 
children, malocclusion about $30 \%$ and oral cancer which accounts for almost 30-35\% of the total diagnosed cancer cases. However, most of the Indian studies have shown that, greatest burden of all these oral problems is on the disadvantaged and socially marginalized people (30).

Dental caries is an ecological disease in which the diet, the host and the microbial flora interact over a period of time in such a way as to encourage demineralization of the tooth enamel with resultant caries formation.
Dental caries is still one of the most common diseases in the world today. Until recently almost everyone had experienced tooth decay in their lifetime. However, today many people are caries free and there has been a 40-60\% reduction in the incidence of tooth decay around the Western world. Most developed countries and many nonindustrialized countries are now well below the World Health Organization goal of less than 3 decayed, missing or filled teeth per 12 year old child (31).

Table.1 Five Major Groups of Viridans Group Streptococci (3)

\begin{tabular}{|l|l|}
\hline Streptococcus mutans group & $\begin{array}{l}\text { Streptococcus mutans, } \\
\text { Streptococcus sorbinus, } \\
\end{array}$ \\
Streptococcus ratti \\
\hline Streptococcus salivarius group & Streptococcus salivarius, \\
& Streptococcus infantarius \\
& Streptococcus vestibularis \\
\hline Streptococcous mitis group & Streptococcus mitis \\
& Streptococcus oralis \\
& Streptococcus infantis \\
& Streptococcus cristatus \\
& Streptococcus perois \\
\hline Streptococcus sanguinis group & Streptococcus sanguinis \\
& Streptococcus parasanguinis \\
& Streptococcus gordonii \\
\hline Streptococcus anginosus group & Streptococcus anginosus \\
& Streptococcus intermedius \\
& Streptococcus constellatus \\
\hline
\end{tabular}

Worldwide, most children and an estimated $90 \%$ of adults have experienced caries, with the disease most prevalent in Latin American countries, countries in the Middle East and South Asia, and least prevalent in China. In the United States, dental caries is the most common childhood disease, being at least five times more common than asthma. It is the primary pathological cause of tooth loss in children.
Between 29 to $59 \%$ of adults over the age of fifty, experience caries. The number of cases has decreased in some developed countries, and this decline is usually attributed to increasingly better oral hygiene practices and preventive measures such as fluoride treatment. Nonetheless, countries that have experienced an overall decrease in case of tooth decay continue to have a disparity in the distribution of the disease. Among 
children in the USA and Europe, $20 \%$ of the population endures 60 to $80 \%$ of cases of dental caries. A similarity skewed distribution of the disease id found throughout the world with some children having none or very few caries and others having a high number. Australia, Nepal and Sweden have a low incidence of cases of dental caries among children whereas cases are more numerous in Costa Rica and Slovakia $(31,32)$.

\section{Prevention of Dental Caries}

Shortly after the introduction of penicillin and, in later years, vancomycin, the notion that dental caries could be treated by antibiotics was tested in laboratory animals. This idea was not pursued, mainly because of the growing fear that pathogenic bacteria may become tolerant to the regular use of sublethal doses of antibiotic. Interestingly, and supportive of the bacterial cause of dental caries, children receiving regular doses of antibiotics for amelioration of symptoms associated with cystic fibrosis exhibited significantly less tooth decay compared with the nonmedicated population. Chlorhexidine was one of the first antiseptic agents proposed for use against dental caries and has proved to be the most effective. Administered by oral lavages or by gels in custom mouthpieces, chlorhexidine effects modest reductions in caries-approximately $30 \%$ in various populations. Because cariogenic bacteria are thought to be transferred from mother to child, several groups have attempted to reduce the levels of cariogenic bacteria so that lesser amounts are transmitted to the infant. The strategy is appealing in that it constitutes primary prevention, attacking the source and nature of the cause (33-35).

Vaccine approaches for eliciting an $\operatorname{IgA}$ or IgG response are being debated. Results using animal models have been promising, especially coupled with newer forms of antigen delivery, such as liposomes, but safety and efficacy in humans have not yet been demonstrated. Whether mutans streptococci alone are the appropriate target, or if other bacteria must be included, is still an open question. Moreover, targeting members of the indigenous flora, as opposed to exogenous pathogens, requires that immunoglobulins distinguish self from nonself antigens so as not to induce an autoimmune reaction. Despite some difficulties that must be overcome, the development of an inexpensive and effective vaccine would be a tremendous tool for combating dental caries, especially in developing countries $(36,37)$.

Tooth extraction is the method of treatment for many individuals, although more expensive restorative therapies involving cleaning of the pulp chamber and filling of the empty canals (i.e.; "root canal") are available. Although the lesion is restored by these approaches, the etiologic agent, that is, the specific bacteria of the plaque biofilm, are left basically intact, available for initiating new lesions should the right environment present. This surgical management of dental caries has been the standard for more than 100 years and constitutes the main approach to the treatment of caries.

Personal hygiene care consists of proper brushing and flossing daily. The purpose of oral hygiene is to minimize any etiologic agents of disease in the mouth. The primary focus of brushing and flossing is to remove and prevent the formation of plaque or dental biofilm. Dietary modification is one of the factors for the prevention of dental caries. For children, the American Dental Association and the European Academy of Paediatric Dentistry recommend limiting the 
frequency of consumption of drinks with sugar, and not giving baby bottles to infants during sleep. Mothers are also recommended to avoid sharing utensils and cups with their infants to prevent transferring bacteria from the mother's mouth. It has been found that milk and certain kinds of cheese like cheddar cheese can help counter tooth decay if eaten soon after the consumption of foods potentially harmful to teeth. Fluoride from small quantities in the drinking water or in the form of other, more concentrated delivery systems, such as mouthwashes, toothpastes, and oral supplements, is well established as effective in reducing caries increment. Also, chewing gum containing xylitol (a sugar alcohol) is widely used to protect teeth in many countries now. Xylitol's effect on reducing dental biofilm is, it is presumed, due to bacteria's inability to utilize it like other sugars (38).

\section{References}

1. Marsh, P.D. 2006. Dental plaque as a biofilm and microbial community implications for health and disease. BMC Oral Health. 6 Suppl. 1: 14.

2. Kajfasz, J. K., I.Rivera-Ramos., J. Abranches., $\quad$ A.R.Martinez., P.L.Rosalen., A.M.Derr., R.G.Quivey and J.A.Lemos. 2010. Two Spx proteins modulate stress tolerance, survival, and virulence in Streptococcus mutans. J Bacteriol. 192: 2546-2556.

3. Jesper Hohwy, Jesper Reinholdt and Mogens Kilian. 2001. Population Dynamics of Streptococcus mitis in Its Natural Habitat. Infection and Immunity. 69(10): 6055 - 6063.

4. Chetan, C., M. Suraj, C. Maheshwari, A. Rahul and P. Priyanka. 2011. Screening of antioxidant activity and phenolic content of whole plant of barleria prionitis linn. Int. J. Res. Ayurveda Pharm. 2: 1313-1319.

5. Bensing, B.A., Rubens, C.E., Sullam, P.M. 2011. Genetic Loci of Streptococcus mitis That Mediate Binding to Human Platelets. Infect Immun. 69, No. 3. p. 1373-1380.

6. Mitchell, J. (2011). Streptococcus mitis: walking the line between commensalism and pathogenesis. Molecular oral microbiology, 26(2), 89-98.

7. Kirchherr, J.L., Bowden, G.H., Richmond, D.A., Sheridan, M.J., Wirth, K.A., Cole, M.F. 2005. Distribution of Streptococcus mitis biovar 1 phenotypes on shedding and non-shedding oral surfaces of human infants during the first year of life. Microbial Ecology in Health and Disease. 17(3): 138 - 145.

8. Nobbs, A. H., Lamont, R. J., \& Jenkinson, H. F. (2009). Streptococcus adherence and colonization. Microbiology and molecular biology reviews: MMBR, 73(3), 407-450.

9. Clarke, J.K. 1924. On the bacterial factor in the aetiology of dental caries. Brit J Exp Pathol. (5):141-147.

10. Loesche, W. J. 1986. Role of Streptococcus mutans in human dental decay. Microbiol. Rev. 50: 353-380.

11. Koneman E.W. 1997.Color Atlas \& Text Book of Diagnostic Microbiology 5th ed Eds. Pub (Lippincott, Williams \& Wilkinth) Philadelphia, 1997, 611-18.

12. Caufield, P.W. 1997. Dental caries--a transmissible and infectious disease revisited: a position paper. Pediatr Dent. 19: 491-8.

13. Tanzer J.M, Baranowski L.K, Rogers J.D, Haase E.M and Scannapieco F.A. 2001. Oral colonization and cariogenicity ofStreptococcus gordonii in specific pathogen-free TAN: SPFOM (OM) BR rats consuming 
starch or sucrose diets. Arch Oral Biol. 46:323-33.

14. Thenisch N.L, Bachmann L.M, Imfeld T, Leisebach Minder T, Steurer and J. Are 2006. mutans streptococci detected in preschool children a reliable predictive factor for dental caries risk? A systematic review. Caries Res 40: 366-374.

15. Berkowitz.R.J. Mutant Streptococci: Acquisition and transmission. Pediartr. Dent. 2006: 28(2): 106-109.

16. Banas J. A. 2004. Virulence properties of Streptococcus mutans. Front. Biosci. 9:1267-1277.

17. Kuramitsu, H.K. 2001. Virulence properties of Oral Bacteria: Impact of Molecular Biology. Curr. Issues $\mathrm{Mol}$ Biol., 3:35-36.

18. Burne. R. A. 1998. Oral streptococci. Products of their Environment; J. Dent. Res. 77.p- 445-452.

19. Giacaman, R.A., Araneda, E., \& Padilla, C. 2001. Association between biofilm-forming isolates of mutans streptococci and caries experience in adults. Archives of Oral Biology. 55.

20. Anderl, J.N.; Franklin, M.J.; Stewart, P.S. Antimicrob. Agents Chemother. 2000, 44, 1818.

21. Baehni, P. C. and Takeuchi, Y. 2003. Anti-plaque agents in the prevention of biofilm-associated oral diseases. Oral Dis., 9:23-9.

22. Taku Fujiwara. 2005. Etiology and Clinical Symptoms of Dental Caries. Foods Food Ingredients J. Jpn. 210: 4.

23. Zaura-Arite E. M.J, ten Cat. 2001. Confocal microscopy study of undisturbed and chlorhexidine-treated dental biofilm. J Dent Res 80: 14361440.

24. Kreth, J., Hagerman, E., Tam, K., Merritt, J., Wong, D. T. W., Wu, B. M., Myung, N. V., Shi, W. \& Qi, F. 2008. Quantitative analyses
ofStreptococcus mutans biofilms with quartz crystal microbalance, microjet impingement and confocal microscopy. Biofilms. 1: 239-263.

25. Banas JA, Vickerman MM. 2003. Glucan-binding proteins of the oral streptococci. Crit Rev Oral Biol Med, 14(2): 89-99.

26. Marsh PD, Nyvad B. 2001. The oral microflora and biofilms in teeth. In: Fejerskov O, Kidd EAM, eds. Dental caries. The disease and its clinical management, 3d edn. Copenhagen: Blackwell Munksgaard. 29-48.

27. Nyvad, B., and M. Kilian. 1990. Comparison of the initial streptococcal microflora on dental enamel in cariesactive and in caries-inactive individuals. Caries Res. 24:267-272.

28. Geddes DAM (1975). Acids produced by human dental plaque metabolism in situ.Caries Res 9:98-109.

29. Geddes DAM (1981). Acids produced by human dental plaque metabolism in situ. Caries Res 9:98-109.

30. WHO Disease and injury country estimates. World Health Organization. 2009. Retrieved Nov 11.

31. The World Oral Health Report 2003. Geneva: World Health Organization.

32. Water Sanitation and Health (WSH), Water related diseases. World Health Organization. Retrieved 22 May 2011.

33. Kohler, B., Andreen, I., and Jonsson, B. 1994. The effect of caries-preventive measures in mothers on dental caries and the oral presence of the bacteria Streptococcus mutans and lactobacilli in their children. Arch Oral Biol .29(11):879-83.

34. Adham M. Abdou, Manal M. E. Ahmed, Yusuke Yamashita, Mujo Kim. 2014. Immunoglobulin: A Natural Way to Suppress Helicobacter pylori in Humans. Scientific research. 781-791. 
35. Wright J.T, Cutter G.R, Dasanayake A.P. 1992. Effect of conventional dental restorative treatment on bacteria in saliva. Community Dent Oral Epidemiol 20:138-143.

36. Michalek SM, Childers NK.1990. Development and outlook for a caries vaccine. Crit Rev Oral Biol Med 1: 3754.

37. Smith, D., and Taubman, M. 1995. Experimental immunization of rats with
Streptococcus mutans 59-kilodalton glucab-binding protein against dental caries. Infect. Immun 64: 3069-3073.

38. Li Xiao -Liang., Shuai jiang - bing, fang Wei-huan.2006. Proteion of Carassius auratus Gibelio against infection by Aeromonas hydrophyla using specific immunoglobulins from hen egg yolk. J.Zhejiang.Univ.Science B. 7(11):922-928.

\section{How to cite this article:}

Dinesh, M.D., M.S. Uma, S. Meenatchisundaram, V.M. Anjali, P.S. Athira and Asitha

Carmel. 2016. Streptococcal Dental Caries - A Short Review. Int.J.Curr.Res.Aca.Rev. 4(1):

160-170. doi: http://dx.doi.org/10.20546/ijcrar.2016.401.015 